\title{
Los predictores de "insight" con respecto a la enfermedad mental entre los pacientes psicóticos y no psicóticos
}

\author{
Andrea Lang, Dr. \\ Gerlinde Berghofer, Dr. \\ Anita Kager, MA \\ Egbert Steiner \\ Margot Schmitz, Dr. \\ Friedrich Schmidl, Dr. \\ Stephan Rudas, MD
}

Servicios Comunitarios de Salud Mental, Viena AUSTRIA

\begin{abstract}
RESUMEN - El propósito de este estudio fue 1) Crear un índice de "insight" en relación con la enfermedad mental 2) Examinar los predictores de "insight" entre una muestra regional representativa compuesta por 418 pacientes.

El "insight" se definió mediante un índice basado en tres cuestiones. Además de estas cuestiones fue tenida en cuenta la estimación hecha por el entrevistador utilizando así mismo un índice. Un buen "insight" o uno "moderado" estaba asociado con una edad joven, estado civil de casado o bien de "conviviente", la existencia de confidente íntimo, el diagnóstico de un trastorno afectivo, de uno ansioso o bien de un trastorno de adaptación, así como de dependencias al alcohol o a otras drogas, baja gravedad de la enfermedad, baja edad del primer tratamiento, alta validación del tratamiento y pocas admisiones durante el periodo de estudio. Utilizando el análisis discriminante se consiguió una clasificación grupal correcta en el $44 \%$ del total de pacientes.

El índice parece ser una medida rápida y aceptable que puede encontrar aplicación como método para medir los diferentes niveles de "insight".
\end{abstract}

\section{Introducción}

Hace ya algunos años los expertos asumieron que el insight con respecto a la enfermedad constituye una dicotomía variable correspondiendo al todo o nada (Van Putten et al.
1976, Lin et al. 1979, Heinrichs et al. 1985). Posteriormente las investigaciones detectaron que el insight comprende una variedad de fenómenos basados en diferentes dimensiones (Wciorka 1988, Greenfield et al. 1989, David, et al. 1990, Amador et al. 1991, 1993) y desarrollados en una escala denominada "Scale 
to Asses Unawareness of Mental Health (SUMD). La SUMD incluye las siguientes dimensiones: Conocimiento de la enfermedad, necesidad de tratamiento médico, conocimiento acerca de las consecuencias sociales de las enfermedades mentales, y conocimiento y atribución de signos específicos.

Los resultados de los estudios sobre el insight no pueden ser comparados entre sí debido al número tan heterogéneo de instrumentos basados en diferentes conceptualizaciones. Algunos estudios han hallado que el funcionamiento social y el insight se encuentran asociados positivamente (Soskis y Bowers 1969, Lysker y Bell 1994, Peralta y Cuesta 1994). McGlashan y Carpenter en 1981 mostraron como el insight correlaciona con el tamaño de la red social. Fennig et al. (1996) encontraron que el insight correlaciona con el estado civil de casado y que los pacientes esquizofrénicos muestran un menor insight que las personas que padecen de trastorno bipolar, depresión mayor u otros trastornos psicóticos. Carpenter et al. (1973) demostraron que el $81 \%$ del total de los esquizofrénicos carecían de insight. David et al. (1992) enfatizaron en el sentido de que no eran solo los esquizofrénicos los que poseían un pobre insight. Amador et al. (1994) también señalaron que las dimensiones del insight de los pacientes con trastorno bipolar eran más bajos al compararlos con los de otros grupos diagnósticos. En la literatura la relación entre insight e impresión clínica global permanece todavía oscura. Los estudios presentan en este sentido resultados inconsistentes. Amador et al. (1991), por ejemplo, señalaron cómo la mayoría de los estudios realizados nunca han examinado la asociación entre psicopatología y nivel de insight. (ver Bartko et al. 1988, McEvoy et al. 1989a 1993). Posteriormente Amador et $a l$. han encontrado cómo el insight correlaciona significativa y positivamente con la gravedad de la psicopatología, aunque la correlación no fue significativa cuando el diagnóstico se revaluó más estrictamente de nuevo. David et al. (1992) observaron que la medida total del insight correlaciona inversamente y moderadamente con la medida global de la psicopatología.

El cumplimiento del tratamiento está asociado positivamente con el insight en los enfermos de esquizofrenia (Lin et al. 1979, Bartko et al. 1988, McEvoy et al. 1989a, 1989b, Lysaker y Bell 1994), mientras que entre los pacientes hospitalizados McEvoy et al. (1989b) no encuentra que el complimiento con la medicación sea un buen predictor del insight. Ellos señalan como incluso una modesta correlación inversa entre el cumplimiento del tratamiento y el insight. Considerando el alta del paciente algunos autores han investigado la asociación positiva con el insight (Lin et al. 1979, McGlashan y Carpenter 1981, Heinrichs et al. 1985, Bartko et al. 1988, McEvoy et al. 1989b). No obstante, algunas de las más tempranas investigaciones parecen no confirmar estos hallazgos (Eskey 1958, Van Putten et al. 1976). Los pacientes con cortas evoluciones de su enfermedad y bajos niveles de insight tuvieron un mayor riesgo de ser rehospitalizados (McEvoy et al. 1993) pero para otros autores el insight no parece ser predictor de nuevas admisiones (Lysaker y Bell 1994).

\section{Metodología}

\section{Sujetos}

Hace más de veinte años, en el curso de una reforma psiquiátrica, en Viena, Austria, se organizó un sistema de asistencia y cuidados de carácter comunitario, sectorizado y coordinado (Rudas 1990). En el mes de 
abril de 1980 se abrió el primero de ocho centros regionales de salud mental. Cada uno de estos ocho se encargaron de un área que comprendía a una población en torno a los 147.000 a 235.000 habitantes.

Los individuos del estudio que nos ocupa eran pacientes externos de cuatro de esos centros comunitarios de salud mental, y también pacientes hospitalizados en dos Unidades, una de ellas en un hospital general, y la otra en un hospital psiquiátrico. La inclusión de paciente comenzó en el mes de enero de 1995 y terminó en abril de 1996. Los criterios de exclusión fueron: no tener voluntad de participar, psicosis muy florida, demencia, deficiencia intelectual e insuficiente conocimiento del idioma alemán. La muestra tuvo en cuenta a pacientes que realizaron el primer contacto con la Institución y a pacientes de larga evolución (que significa que la duración del tratamiento como pacientes externos había sido de más de tres meses, y que los pacientes internados de larga evolución habían realizado al menos dos admisiones o ingresos).

La muestra total estaba formada por 448 pacientes. De ellos un total de 23 enfermos no se incluyeron porque no terminaron la primera entrevista. No se encontraron diferencias significativas entre los pacientes incluidos y los excluidos en términos como sus características demográficas y clínicas. La población final del estudio quedó compuesta por un total de 425 pacientes. Todos ellos dieron su consentimiento escrito a la entrevista. Al comienzo de la investigación 165 (39\%) se hallaban hospitalizados, 202 (48\%) eran pacientes de primer contacto con ellos y $223(52 \%)$ eran pacientes de larga evolución. La inclusión de estos últimos se realizó randomizados en seis días. A los pacientes de primer contacto se les preguntó si querían participar en la secuencia de su aparición durante el periodo de inclusión del estudio.

\section{Instrumentos y colección de los datos}

Se construyó una entrevista semi-estandarizada que recoge las características demográficas y clínicas, así como las informaciones relacionadas con los tratamientos. El status clínico de los pacientes y sus condiciones en cuanto a sus habilidades se recogieron mediante la Global Assessment of Functioning Scale (GAF), que es una revisión de la GAS (Endicott et al. 1976) y de la CGAS (Shaffer et al. 1978). La gravedad general del paciente se midió mediante la Clinical Global Impresión Scale (CGI) (National Institute of Mental Health 1976). El nivel de salud mental y física se recogió utilizando una escala visual análoga de $100 \mathrm{~mm}$. El pronóstico se consideró como pesimista cuando la diferencia era menor de 10 puntos, cero o negativa. Fueron éstas aplicadas por clínicos y auto-aplicadas por los propios pacientes.

Para este estudio se desarrolló un nuevo índice para la medición del insight en relación con la enfermedad mental, basado éste en cuatro ítems. A cada uno de los ítems se les asignó un punto: si el paciente estaba de acuerdo en el hecho de sentirse enfermo, si el paciente era capaz de nominar el diagnóstico correcto y/o los síntomas, si el paciente tenía al menos una expectativa terapéutica. En relación con las expectativas terapéuticas, se les preguntaba a los pacientes si ellos sentían que sus síntomas, su situación laboral, su situación en cuanto al hogar, contactos sociales, quehaceres domésticos diarios, así como la estabilidad de su estado de salud había mejorado en relación con el tratamiento. Adicionalmente, la importancia de las expectativas en cuanto al tratamiento fueron evaluadas dándoles el peso de un punto, solamente cuando el paciente entrevistado considerase estas expectativas terapéuticas como importantes o tal vez impor- 
tantes. Además se asignó un punto en el caso de que el entrevistador valorase al paciente como que posee insight.

De acuerdo con la suma de puntos se definieron cuatro niveles de insight: buen insight (4 puntos), insight moderado (3 puntos), pobre insight (2 puntos) у mиy pobre insight (1 punto o 0 puntos).
Los entrevistadores fueron psicólogos que tenían suficiente experiencia en el trabajo con pacientes psiquiátricos. Por otro lado los entrevistadores se mantuvieron independientes de los tratamientos seguidos por los pacientes. Antes del estudio los entrevistadores pasaron con éxito por la debida calibración de sus valoraciones. El trabajo estadístico se llevó a cabo utilizando el paquete

Tabla I

Rasgos demográficos (N=418)

Características

n

\begin{tabular}{|c|c|c|}
\hline \multicolumn{3}{|l|}{ Edad } \\
\hline media (años) & 44,0 & $15,2(\mathrm{SD})$ \\
\hline \multicolumn{3}{|l|}{ Sexo } \\
\hline hombre & 222 & 53 \\
\hline mujer & 196 & 47 \\
\hline \multicolumn{3}{|l|}{ Estado civil } \\
\hline soltero & 167 & 40 \\
\hline casado/cohabitante & 109 & 26 \\
\hline divorciado/separado & 117 & 28 \\
\hline viudo & 25 & 6 \\
\hline \multicolumn{3}{|l|}{ Empleo } \\
\hline sí & 92 & 22 \\
\hline no & 325 & 78 \\
\hline \multicolumn{3}{|l|}{ Educación profesional } \\
\hline terminada & 276 & 66 \\
\hline no terminada & 142 & 34 \\
\hline \multicolumn{3}{|l|}{ Diagnóstico (DSM-IV) } \\
\hline trastornos orgánicos & 19 & 5 \\
\hline dependencia a alcohol o drogas & 80 & 19 \\
\hline trastornos esquizofrénicos & 168 & 40 \\
\hline trastornos del humor & 53 & 13 \\
\hline trastornos de ansiedad de adaptación & 62 & 15 \\
\hline trastornos de personalidad & 34 & 8 \\
\hline \multicolumn{3}{|l|}{ Admisiones previas } \\
\hline 0 & 140 & 34 \\
\hline 1 & 68 & 16 \\
\hline $2-5$ & 122 & 29 \\
\hline 6 y más & 88 & 21 \\
\hline \multicolumn{3}{|l|}{ Duración enfermedad } \\
\hline media (años) & 8,8 & $10,9(\mathrm{SD})$ \\
\hline \multicolumn{3}{|l|}{ Insight } \\
\hline muy pobre & 67 & 16 \\
\hline pobre & 81 & 19 \\
\hline moderado & 133 & 32 \\
\hline bueno & 137 & 33 \\
\hline \multicolumn{3}{|l|}{ Insight de pacientes no psicóticos } \\
\hline muy pobre & 20 & 12 \\
\hline pobre & 24 & 15 \\
\hline moderado & 66 & 41 \\
\hline bueno & 51 & 32 \\
\hline
\end{tabular}


estadístico SPSS 6.1.3. Los datos fueron analizados utilizando los tests ANOVA de una vía y Chi cuadrado. El análisis discriminante se realizó con el fin de determinar las características del paciente relacionadas con el insight en relación con la enfermedad.

\section{Resultados}

\section{Aspectos demográficos}

Siete pacientes de entre el total de la muestra fueron clasificados como "no enfermos" de acuerdo con la escala CGI. Así que del total de la muestra $(\mathrm{N}=425)$ fueron datos válidos los provenientes de 418 personas a fin de proceder a la valoración del insight. Las características demográficas de la muestra se presentan en la tabla I.

El 53\% de los individuos eran hombres. Los pacientes fueron diagnosticados por los clínicos de acuerdo con los criterios diagnósticos emanados de la DS-IV. Casi la mitad de los pacientes sufrían de esquizofrenia (40\%). El 20\% no eran realmente pacientes psicóticos, tratándose por ejemplo de trastornos de ansiedad, de adaptación o de dependencias a alcohol o a drogas. La muestra tenía una edad media de 44 años con un SD de 15,2 años. El $40 \%$ eran solteros. Más de la tercera parte $(34 \%)$ de todos ellos no habían terminado su formación educativa profesional y el $78 \%$ se hallaban en situación de desempleo. La duración de la enfermedad en el 37\% de los casos había sido de dos años, y en el $31 \%$ la duración era mucho mayor, de más de 10 años. El 34\% de los pacientes nunca había sido hospitalizado, el $16 \%$ había hecho únicamente un ingreso, el 29\% había hecho de dos a cinco ingresos, y el $21 \%$ del total había tenido más de seis ingresos. La mayor parte de los pacientes $(65 \%)$ mostraron un moderado o un buen insight. Veinte pacientes
(12\%) de la muestra "no psicótica" $(\mathrm{N}=$ 161) mostraron poseer "muy pobre insight".

\section{Características demográficas, anamnésicas y del tratamiento, niveles de "insight"}

La distribución demográfica y las características propias de la anamnesis correspondientes a los cuatro niveles del insight aparecen presentadas en las tablas II y III.

Los resultados obtenidos muestran la no existencia de asociación entre el insight y la pertenencia a un sexo determinado (tabla II). Los pacientes más jóvenes en el periodo de estudio y cuando utilizaron los cuidados psiquiátricos por vez primera, mostraron de modo significativo mayor insight que los mayores de edad (tabla III). El estado civil y/o el poseer un confidente íntimo fue discriminativo para los dos grupos (tabla II). El estado civil casado/cohabitante estaba fuertemente correlacionado con un "moderado insight" (tabla II). Los pacientes viudos mostraron de modo significativo más a menudo "muy pobre insight". Los pacientes con "muy pobre insight" generalmente no poseían confidentes íntimos (tabla II). Los pacientes con esquizofrenia o con trastornos de tipo orgánico demostraron básicamente "pobre insight" (tabla II). Las personas que padecían de dependencias al alcohol o a drogas mostraron de modo significativo más a menudo "insight moderado". La apariencia de un "buen insight' fue más alta entre los pacientes con trastornos de tipo afectivo, ansioso o de adaptación. Los pacientes cuya gravedad de su enfermedad medida mediante la CGI, fue evaluada como incierta o no importante, mostraron significativamente más a menudo "insight moderado" o "bueno" (tabla II). "Muy pobre insight" se asociaba con alto nivel de salud física y mental auto-evaluada por el propio paciente (tabla III). El funcionamiento global 
Tabla II

Asociación entre el Insight y las características demográficas y anamnésicas

\begin{tabular}{|c|c|c|c|c|c|c|c|}
\hline Variable & $\begin{array}{c}\text { Muy pobre } \\
\text { Insight } \\
\mathrm{n}=67 \\
\%\end{array}$ & $\begin{array}{c}\text { Pobre } \\
\text { Insight } \\
\mathrm{n}=81 \\
\%\end{array}$ & $\begin{array}{c}\text { Moderado } \\
\text { Insight } \\
\mathrm{n}=133 \\
\%\end{array}$ & $\begin{array}{c}\text { Bueno } \\
\text { Insight } \\
\mathrm{n}=137 \\
\%\end{array}$ & $\chi^{2}$-test & df & $\begin{array}{l}\text { signo } \\
\text { valor } \\
\text { de la p* }\end{array}$ \\
\hline \multicolumn{8}{|l|}{ Sexo } \\
\hline hombre & 55 & 48 & 59 & 49 & \multirow[t]{2}{*}{$\chi^{2}=4,0$} & \multirow[t]{2}{*}{3} & \multirow[t]{2}{*}{ n.s. } \\
\hline mujer & 45 & 52 & 41 & 51 & & & \\
\hline \multicolumn{8}{|l|}{ Estado civil } \\
\hline soltero & 35 & 45 & 41 & 39 & \multirow{4}{*}{$\chi^{2}=35,1$} & \multirow[t]{4}{*}{9} & \multirow[t]{4}{*}{$<, 001$} \\
\hline casado/cohabitante & 12 & 25 & 33 & 28 & & & \\
\hline divorciado/separado & 33 & 26 & 24 & 29 & & & \\
\hline viudo & 20 & 4 & 2 & 4 & & & \\
\hline \multicolumn{8}{|l|}{ Confidente íntimo } \\
\hline sí & 73 & 77 & 86 & 88 & \multirow{2}{*}{$\chi^{2}=9,5$} & \multirow[t]{2}{*}{3} & \multirow[t]{2}{*}{, 023} \\
\hline no & 27 & 23 & 14 & 12 & & & \\
\hline \multicolumn{8}{|l|}{ Diagnostico (DSM) } \\
\hline trastornos orgánicos & 9 & 7 & 3 & 2 & \multirow{6}{*}{$\chi^{2}=33,7$} & \multirow[t]{6}{*}{15} & \multirow{6}{*}{, 004} \\
\hline dependencia a alcohol o drogas & 16 & 15 & 28 & 15 & & & \\
\hline trastornos esquizofrénicos & 51 & 52 & 32 & 37 & & & \\
\hline trastornos del humor & 6 & 12 & 12 & 18 & & & \\
\hline trastornos de ansiedad de adaptació & ión 5 & 8 & 20 & 20 & & & \\
\hline trastornos de personalidad & 13 & 6 & 7 & 8 & & & \\
\hline \multicolumn{8}{|l|}{ Gravedad de la enfermedad (CGI) } \\
\hline incierto & 6 & 3 & 9 & 2 & \multirow[t]{5}{*}{$\chi^{2}=28,7$} & \multirow[t]{5}{*}{15} & \multirow[t]{5}{*}{, 017} \\
\hline ligero & 6 & 5 & 6 & 16 & & & \\
\hline moderado & 16 & 28 & 28 & 20 & & & \\
\hline definitely & 46 & 44 & 38 & 41 & & & \\
\hline grave/extremo & 26 & 20 & 19 & 21 & & & \\
\hline \multicolumn{8}{|c|}{ Resultado** (autoevaluado por el paciente) } \\
\hline optimista & 70 & 73 & 72 & 75 & \multirow[t]{2}{*}{$\chi^{2}=, 59$} & \multirow[t]{2}{*}{3} & \multirow[t]{2}{*}{ n.s. } \\
\hline pesimista & 30 & 27 & 28 & 25 & & & \\
\hline \multicolumn{8}{|l|}{ Resultado** (evaluado por el clínico) } \\
\hline optimista & 28 & 41 & 36 & 40 & \multirow{2}{*}{$\chi^{2}=3,2$} & \multirow[t]{2}{*}{3} & \multirow[t]{2}{*}{ n.s. } \\
\hline pesimista & 72 & 59 & 64 & 60 & & & \\
\hline
\end{tabular}

Valor de la p* para los diferentes subgrupos.

n.s.: no significativo.

Resultado** (expectativas en un año: estado de la salud física y mental).

(GAF) (tabla III), el nivel de salud física y mental evaluada por el clínico (tabla III) y el optimismo (pesimismo) de los pacientes y los clínicos en relación con el alta del paciente (tabla II) no parecieron tener influencia.

La distribución de los factores relacionados con el tratamiento seguido teniendo en cuenta los cuatro niveles del insight, aparecen en la tabla IV.
El tiempo y duración del contacto habido (primer contacto o largos contactos en relación con los servicios seleccionados), el cumplimiento terapéutico, la duración del tratamiento y las admisiones previas no mostraron influencia en cuanto a los cuatro niveles del insight de los pacientes. No obstante ello, los pacientes con "pobre insight" de forma significativa realizaron más a menudo al menos un ingreso a lo largo de la duración 
Tabla III

Asociaciones entre "insight", características demográficas, autonomía (GAF) y salud física y mental

\begin{tabular}{|c|c|c|c|c|c|c|c|c|c|}
\hline \multirow[b]{2}{*}{ Variable } & \multicolumn{2}{|c|}{$\begin{array}{c}\text { Muy pobre } \\
\text { Insight } \\
\mathrm{n}=67\end{array}$} & \multicolumn{2}{|c|}{$\begin{array}{l}\text { Pobre } \\
\text { Insight } \\
\mathrm{n}=81\end{array}$} & \multicolumn{2}{|c|}{$\begin{array}{c}\text { Moderado } \\
\text { Insight } \\
\mathrm{n}=133\end{array}$} & \multicolumn{2}{|c|}{$\begin{array}{c}\text { Buen } \\
\text { Insight } \\
\mathrm{n}=137\end{array}$} & \multirow{2}{*}{$\begin{array}{r}\text { Valor } \\
\text { de la p* } \\
\text { signo }\end{array}$} \\
\hline & media & $\mathrm{SD}$ & media & SD & media & $\mathrm{SD}$ & media & SD & \\
\hline Edad en el periodo del estudio & 50,1 & 19,7 & 45,8 & 16,6 & 42,2 & 12,2 & 41,8 & 13,6 & $<, 001$ \\
\hline Edad en el primer tratamiento & 40,2 & 21,2 & 37,0 & 15,9 & 32,8 & 13,2 & 32,7 & 15,6 & ,004 \\
\hline Funcionamiento global (GAF) & 46,9 & 16,0 & 49,7 & 15,9 & 52,8 & 16,7 & 49,7 & 15,9 & n.s. \\
\hline Salud (autoevaluada por paciente) & 64,8 & 24,1 & 59,3 & 27,9 & 59,4 & 23,4 & 44,5 & 19,7 & $<, 001$ \\
\hline Salud (evaluada por el clínico) & 50,7 & 19,8 & 50,3 & 18,0 & 54,8 & 18,3 & 54,0 & 19,6 & n.s. \\
\hline
\end{tabular}

Valor de la $\mathrm{p}^{*}$ para diferencias entre subgrupos.

La significación se probó con el ANOVA de una vía.

n.s.: no significativo.

Tabla IV

Asociaciones entre el "insight" y las variables relacionadas con el tratamiento

\begin{tabular}{|c|c|c|c|c|c|c|c|}
\hline Variable & $\begin{array}{c}\text { Muy pobre } \\
\text { Insight } \\
\mathrm{n}=67 \\
\%\end{array}$ & $\begin{array}{c}\text { Pobre } \\
\text { Insight } \\
\mathrm{n}=81 \\
\%\end{array}$ & $\begin{array}{c}\text { Moderado } \\
\text { Insight } \\
\mathrm{n}=133 \\
\%\end{array}$ & $\begin{array}{c}\text { Buen } \\
\text { Insight } \\
\mathrm{n}=137 \\
\%\end{array}$ & $\chi^{2}$-Test & $\mathrm{df}$ & $\begin{array}{l}\text { signo } \\
\text { valor } \\
\text { de la p* }\end{array}$ \\
\hline \multicolumn{8}{|l|}{ Contacto } \\
\hline pacientes en el primer contacto & 46 & 46 & 45 & 52 & \multirow[t]{2}{*}{$\chi^{2}=1,5$} & \multirow[t]{2}{*}{3} & \multirow[t]{2}{*}{ n.s. } \\
\hline pacientes de larga evolución & 54 & 54 & 55 & 48 & & & \\
\hline \multicolumn{8}{|l|}{ Cumplimiento terapéutico } \\
\hline cumplimiento & 94 & 85 & 91 & 94 & \multirow{3}{*}{$\begin{array}{c}\chi^{2}=14,8 \\
6\end{array}$} & \multirow[t]{2}{*}{12} & \multirow[t]{2}{*}{ n.s. } \\
\hline no cumplimiento & & 6 & 15 & 9 & & & \\
\hline \multicolumn{7}{|l|}{ Duración del tratamiento } & \\
\hline$<2$ años & 39 & 39 & 30 & 34 & \multirow[t]{4}{*}{$\chi^{2}=7,6$} & \multirow[t]{4}{*}{12} & \multirow{4}{*}{ n.s. } \\
\hline $2-5$ años & 16 & 16 & 22 & 22 & & & \\
\hline 6-10 años & 13 & 16 & 12 & 16 & & & \\
\hline$>10$ años & 32 & 29 & 36 & 28 & & & \\
\hline \multicolumn{8}{|l|}{ Admisiones previas } \\
\hline 0 & 30 & 32 & 34 & 36 & \multirow[t]{4}{*}{$\chi^{2}=4,1$} & \multirow[t]{4}{*}{9} & \multirow[t]{4}{*}{ n.s. } \\
\hline 1 & 16 & 15 & 16 & 17 & & & \\
\hline $2-5$ & 26 & 35 & 29 & 28 & & & \\
\hline 6 o más & 28 & 18 & 21 & 19 & & & \\
\hline \multicolumn{8}{|l|}{ Admisiones durante el estudio } \\
\hline 0 & 50 & 42 & 70 & 58 & \multirow[t]{2}{*}{$\chi^{2}=10,4$} & \multirow[t]{2}{*}{3} & \multirow[t]{2}{*}{, 015} \\
\hline 1 o más & 50 & 58 & 30 & 42 & & & \\
\hline \multicolumn{8}{|l|}{ Coste del tratamiento } \\
\hline 0-100 öS & 67 & 62 & 41 & 38 & \multirow[t]{3}{*}{$\chi^{2}=22,9$} & \multirow[t]{3}{*}{6} & \multirow[t]{3}{*}{$<, 001$} \\
\hline 101-500 öS & 20 & 30 & 35 & 41 & & & \\
\hline >500 öS & 13 & 8 & 24 & 21 & & & \\
\hline
\end{tabular}

Valor de la p* para diferencias entre subgrupos. n.s.: no significativo. 
de esta investigación. Los pacientes con "insight moderado" o "bueno" valoraron su tratamiento de forma más alta al compararlos con aquellos otros pacientes que ofrecían "muy pobre insight" o "pobre insight".

Mediante el análisis discriminante (los datos no aparecen en la tabla) los siguientes factores fueron utilizados como variables dependientes: edad, sexo, estado civil, confidente íntimo, edad en el inicio del primer tratamiento, diagnóstico, CGI, GAF, cumplimiento terapéutico, tipo de asistencia (ambulatoria o en régimen de hospitalización), estado de salud auto-evaluado por el propio paciente, estado de salud evaluada por el clínico, optimismo en relación con el alta, primer contacto/larga evolución del paciente, validación del tratamiento y efectos secundarios de la medicación. El insight con relación a la enfermedad padecida fue utilizado como la variable de clase. De acuerdo con el análisis se dieron tres coeficientes discriminantes, tales como la edad (Wilk's lambda 0,83, p < 0,001), estado de salud física y mental auto-evaluada (Wilk's lambda $0,89, \mathrm{p}<0,001, \mathrm{y}$ validación del tratamiento (Wilk's lambda 0,79, $\mathrm{p}<0,001$ ) diferenciaron de modo significativo los cuatro grupos de insight. Se consiguió así una clasificación agrupada de modo correcto del $44 \%$ en comparación con la selección natural de solo el $25 \%$. El porcentaje de asignación correcta a un grupo fue altamente relacionada con el "buen insight" (68\%), seguida por "moderado insight" (42\%) y "pobre insight" (27\%). Solamente un porcentaje del $15 \%$ se asignó al grupo de "muy poco insight".

\section{Discusión}

Esta investigación se hizo con el fin de conseguir un índice para la medida del insight con relación a la enfermedad mental. De forma especial, para los clínicos este índice puede ser interesante debido a la sencillez de su manejo y porque además es muy corto. Adicionalmente, la revisión de la literatura más relevante prueba cómo las dimensiones elegidas (conocimiento de estar enfermo, conocimiento de la enfermedad y sus síntomas, expectativas con respecto al tratamiento, evaluación del insight por parte del entrevistador) son válidas para poder llegar a evaluar el insight. No obstante ello, los hallazgos necesitan ser interpretados teniendo en cuenta las limitaciones propias del estudio realizado. Son necesarios estudios posteriores para determinar la validez y fiabilidad del índice. Uno de los principales problemas en estas investigaciones es que los resultados no pueden ser comparados con los de otras investigaciones dada la gran cantidad de medidas heterogéneas basadas en muy diferentes conceptualizaciones como existen. Para mejorar la comparabilidad sería necesario un instrumento consensuado para la medida del insight que está por desarrollar.

Nosotros hemos encontrado que el insight se corresponde con diferentes factores de índole demográfico, anamnésico y relacionados con los tratamientos seguidos. Este trabajo constituye una contribución significativa para el conocimiento del insight dada la magnitud de las muestras estudiadas tanto de pacientes psicóticos como de no-psicóticos. Así el insight pobre lo hemos hallado en tres grupos diferentes: 1) En pacientes con pocos contactos sociales, 2) En pacientes con esquizofrenia o con trastornos orgánicos, y 3) En pacientes que consideraban su enfermedad física y mental como menos grave mientras que los clínicos la consideraban más grave.

Por lo que sabemos el insight de los pacientes psiquiátricos no-psicóticos no se ha estudiado demasiado. No obstante ello el $12 \%$ del total de nuestros pacientes no-psicóticos mos- 
traron muy poco insight; el $11 \%$ de estos pacientes no-psicóticos sufrían de dependencia alcohólica o a otras drogas, el $6 \%$ poseían trastornos orgánicos y el 3\% sufrían de trastornos de ansiedad o de adaptación. El "muy poco insight" se encontró entre los pacientes con trastornos orgánicos. Diferentes estudios han mostrado cómo los esquizofrénicos poseen un pobre insight siendo éste causado por la afectación cognitiva concomitante con la enfermedad (Fennig et al. 1996, Lysaker y Bell 1994, McEvoy et al. 1996). La pérdida de la capacidad cognitiva en el caso de los trastornos orgánicos soporta igualmente esta interpretación. Los pacientes con "muy pobre" o "pobre insight" eran significativamente mayores en el momento del primer tratamiento psiquiátrico. Para explicar ello debe ser tenido en cuenta el diagnóstico. Los pacientes con trastornos orgánicos eran a menudo mayores en su primer tratamiento y ellos mostraron menos insight. El 55\% de los pacientes con esquizofrenia tenían "muy pobre" o "pobre insight". La comparación entre todos los diagnósticos demostró como el "pobre insight" estaba significativamente asociado con la esquizofrenia (ver también Fennig et al. 1996, Carpenter et al. 1973). En contraste con Amador et al. (1994) nosotros encontramos que el diagnóstico de trastorno del humor se relaciona con un "buen insight". En la mayoría de los casos no fue posible entrevistar a los pacientes maníacos. No obstante ello, en el curso de los episodios maníacos el insight de los pacientes declina. Son necesarios estudios futuros que se centren en el estudio de los cambios del insight de acuerdo con los diferentes estadios de la enfermedad. Los pacientes con dependencias al alcohol o a otras drogas poseían los más altos niveles de insight. La motivación terapéutica podría explicar este resultado. La rehabilitación de los pacientes dependientes del alcohol o de las drogas podría fallar en el caso de una falta de motivación al comenzar el pro- grama de rehabilitación. Es obvio que el insight en relación con la enfermedad mental y la motivación terapéutica están asociadas.

Teniendo en cuenta los ingresos o admisiones de los pacientes, nosotros asumimos el hecho de que el insight se halla asociado con unos resultados favorables en el curso de los seguimientos de estos enfermos. Esta idea fue verificada y los resultados se hallan confirmados por la literatura (Lin et al. 1979, McGlashan y Carpenter 1981, Heinrichs et al. 1985, Bartko et al. 1988, McEvoy et al. 1989b) En contraste con otros (Lysaker y Bell 1994, Bartko et al. 1988, Lin et al. 1979, McEvoy et al. 1989a , 1989b) no se encontró ninguna asociación entre cumplimiento terapéutico e insight. Presumiblemente las respuestas sociales positivas sean la causa de muy diferentes resultados. Solamente el $8 \%$ de nuestra muestra randomizada confesó ignorar la medicación prescrita. No obstante otros estudios hablan de cómo el cumplimiento terapéutico se sitúa entre el $25 \%$ y el 50\% (Boyd et al. 1974, Blackwell 1973, Buzón y Dunn 1986, Sackett y Snow 1979).

El análisis discriminante muestra que las edades más jóvenes, los pacientes con baja auto-evaluación de su salud mental y física y con alta valoración de su tratamiento (económico) son predictores de "buen insight". No obstante la adaptación del modelo discriminante no es en cierto sentido satisfactorio, sugiriendo que otras variables diferentes puedan diferenciar mejor entre los niveles del insight ya que el grupo correcto de clasificación alcanzó solamente al 44\% de los pacientes.

Nuestros resultados indican que un pequeño número de pacientes no psicóticos y también algunos de los que padecían de trastornos orgánicos estaban faltos de insight. De todos modos el insight pobre se hallaba positivamente asociado con el padecimiento de una 
enfermedad grave y con ingresos hospitalarios durante el periodo de estudio. El conocimiento de los pacientes acerca de su diagnóstico, psicopatología y resultados deberían ser mejorados. El ánimo, la esperanza y el conocimiento acerca de la enfermedad combinado ello con la lucha frente a los prejuicios negativos podrían influenciar el cumplimiento de los tratamientos, la calidad de vida y en último término pero no lo menos importante el insight. Todo ello puede abrir la posibilidad de una mejora en las condiciones de vida de los pacientes con enfermedad mental.

\section{Bibliografía}

AMADOR, X.F., STRAUSS, D.H., YALE, S.A. et al. Awareness of illness in schizophrenia. Schizophrenia Bulletin, 17, 1 13-132, 1991.

AMADOR, X.F., STRAUSS, D.H., YALE, S.A. et al. Assessment of insight in psychosis. American Journal of Psychiatry, 150, 873-879, 1993.

AMADOR, X.F., STRAUSS, D.H., YALE, S.A. et al. Awareness of illness in schizophrenia and schizoaffective and mood disorders. Archives of General Psychiatry, 51, 826-836, 1994.

BARTKO, G., HERCZOG, I., ZADOR, G. Clinical symptomatology and drug compliance in schizophrenic patients. Acta Psychiatrica Scandinavica, 77, 74-76, 1988.

BLACKWELL, B. Treatment adherence. British Journal of Psychiatry, 129, 513-531, 1976.

BOYD, J., COVINGTON, T., STANASZEK, W. Drug defaulting, II: analysis of compliance patterns. American Journal Hospital Pharmacy, 31, 485-491, 1974.

BUSSON, M., DUNN, A. Patients' knowledge about prescribed medicines. Pharmaceutical Journal, 236, 624626, 1986.

CARPENTER, W.T. Jr., STRAUSS, J.S., BARTKO, J.J. Flexibel systems for the diagnosis of schizophrenia. Report from the World Health Organisation International Pilot Study of Schizophrenia. Science, 182, 1275-1278, 1973.

COLLINS, A., REMINGTON, G., COULTER, K. et al. Insight, neurocognitive function and symptom clusters in chronic schizophrenia. Schizophrenia Research, 27, 37-44, 1997.

CUESTA, M., PERALTA, V. Lack of insight in schizophrenia. Schizophrenia Bulletin, 20, 359-366, 1994.

DAVID, A.S., BUCHANAN, A., REED, A. et al. The assessment of insight in psychosis. British Journal of Psychiatry, 161, 599-602, 1992.

DAVID, A.S. On insight and psychosis: discussion paper. The Journal of the Royal Society of Medicine, 83, 325-329, 1990.

ENDICOTT, J., SPITZER, R.L., FLEISS, R.L. et al. The Global Assessment Scale: A procedure for measuring overall severity of psychiatric disturbance. Archives of General Psychiatry, 33, 766-771, 1976.

ESKEY, A. Insight and prognosis. Journal of Clinical Psychology, 14, 426-429, 1958.

FENNIG, S., EVERETT, E., BOMET, E.J. et al. Insight in first-admission psychotic patients. Schizophrenia Research, 22, 257-263, 1996.

GREENFIELD, D., STRAUSS, J.S., BOWERS, M.B. et al. Insight and interpretation of illness in recovery from psychosis. Schizophrenia Bulletin, 15, 245-252, 1989.

HEINRICHS, D.W., COHEN, B.P., CARPENTER, W.T. Early insight and the management of schizophrenic decompensation. Journal of Nervous and Mental Disease, 173, 133-138, 1985.

LIN, I.F., SPIGA, R., FORTSCH, W. Insight and adherence to medication in chronic schizophrenics. Journal of Clinical Psychiatry, 40, 430-432, 1979.

LYSAKER, P.H., BELL, M.D. Insight and cognitive impairment. Journal of Nervous and Mental Disease, 182, 656-660, 1994.

MARKOVÁ, I., BERRIOS, G. Insight in clinical psychiatry revisited. Comprehensive Psychiatry, 5, 367-376, 1995.

MARKOVÁ, I., BERRIOS, G. The assessment of insight in clinical psychiatry: a new scale. Acta Psychiatrica Scandinavica, 86, 159-164, 1992.

MCEVOY, J.P., ALAND, J., WILSON, W.H. et al. Measuring chronic schizophrenic patients' attitudes toward their illness and treatment. Hospital and Community Psychiatry, 32, 856-858, 1981.

MCEVOY, J.P., APPELBAUM, P.S., GELLER, L.J. et $a l$. Why must some schizophrenic patients be involuntarily committed? The role of insight. Comprehensive Psychiatry, 10, 13-17, 1989a. 
MCEVOY, J.P., APPERSON, L.J., APPELBAUM, P.S. et al. Insight in schizophrenia. Its relationship to acute schizophrenia. Journal of Nervous and Mental Disease, 177, 43-47, 1989b.

MCEVOY, J.P., FRETER, S., MERRIT, M. et al. Insight about psychosis among outpatients with schizophrenia. Hospital and Community Psychiatry, 44, 883884, 1993.

MCEVOY, J.P., HARTMAN, M., GOTTLIEB, D. et al. Common sense, insight, and neuropsychological test performance in schizophrenia patients. Schizophrenia Bulletin, 22, 635-641, 1996.

MCGLASHAN, T.H., CARPENTER, W. Jr. Does attitude toward psychosis relate to outcome? American Journal of Psychiatry, 138, 797-801, 1981.

National Institute of Mental Health. 028. CGI Clinical Global Impressions. In: GUY W., BONATO R.R., eds. ECDEU Assessment Manual for Psychopharmacology Rev. ed. Rockville, Maryland, 217-222, 1976.

PERALTA, V., CUESTA, M. Lack of insight: Its status within schizophrenic psychopathology. Society of Biological Psychiatry, 36, 559-561, 1994.

RUDAS, S. On measuring the changes in psychiatric care systems: results in an urban area (Vienna). Psychiatric Bulletin, 14, 262-266,1990.

SACKETT, D., SNOW, J. The magnitude of compliance and noncompliance. In: HAYNES, R., TAYLOR, D.,
SACKETT, D., eds. Compliance and Health Care. Baltimore: Journal Hopkins University Press, 1979.

SHAFFER, D., GOULD, M.S., BRASIC, J., et al. Children's Global Assesessment Scale [CGAS]. Archives of General Psychiatry, 40, 1228-1231, 1978.

SMITH, T.E., HULL, J.W., GOODMAN, M. et al. The relative influences of symptoms, insight, and neurocognition on social adjustment in schizophrenia and schizoaffective disorder. The Journal of Nervous and Mental Disease, 187, 102-108, 1999.

SOSKIS, D.A., BOWERS, M.B. The schizophrenic experience: A follow-up study of attitude and post hospital adjustment. Journal of Nervous and Mental Disease, 149, 443-449, 1969.

TOWNSEND, J.M. Cultural conceptions and mental illness: a controlled comparison of Germany and America. Journal of Nervous and Mental/Disease, 160, 409-421, 1975.

VAN PUTTEN, T. CRUMPTON, E, YALE C. Drug refusal in schizophrenia and the wish to be crazy. Archives of General Psychiatry, 33, 1443-1446, 1976.

Dirección para correspondencia:

Dr. Andrea Lang

E-mail: psd-quasi@netway.at

Community Mental Health Services

Marianneng. 1/15

A-1090 Vienna

AUSTRIA 